\title{
TEST OF EMPIRICAL SEDIMENT TRANSPORT RELATIONS AGAINST EXPERIMENTAL SWASH DATA UNDER THE NON-CAPACITY MODELING FRAMEWORK
}

Peng Hu, Institute of Port, Coastal and Nearshore Engineering, Zhejiang University, pengphu@zju.edu.cn Liming Tan, Institute of Port, Coastal and Nearshore Engineering, Zhejiang University, tanliming@zju.edu.cn

Jiafeng Xie, Institute of Port, Coastal and Nearshore Engineering, Zhejiang University, hanbagui@vip.qq.com Zhiguo He, Institute of Port, Coastal and Nearshore Engineering, Zhejiang University, hezhiguo@zju.edu.cn

\section{ABSTRACT}

Swash sediment transport and beach deformation has received great attention in the past two decades. Quantification of swash-induced sediment transport rate is of vital importance for accurate prediction of beach deformation in the swash zone. Two empirical parameters are involved in this quantification, empirical relations for sediment transport capacity and the bed shear stress that may be used in the former.

Since the swash zone is highly unsteady, of short crossshore distance, sediment transport in this zone may be of high possibility to be lag of the flow variation. Thus we have firstly developed a non-capacity sediment transport model for the swash zone. This model appreciates the fact that the actual sediment transport rate may not be necessarily equal to the sediment transport capacity of the flow. In contrast to traditional capacity models that calculate sediment transport rate using directly empirical relations (Hu et al. 2015), the non-capacity model uses the advection-diffusion equation to calculate depthaveraged sediment concentration firstly, and afterwards compute sediment transport rate as flow depth*velocity*concentration. We have also noted that some empirical relations for sediment transport capacity may predict physically unrealistic high values of sediment concentration in the swash zone. This is attributed to the vanishing water depth in the swash zone, whereas existing empirical relations are developed for relatively large water depths ( $\mathrm{Hu}$ et al. 2015; Li et al. 2017).

Experimental swash flows from an Aberdeen flume are numerically simulated. A 3D Reynolds-averaged NavierStokes (RANS) numerical model is applied to the dambreak flow-induced swash flows to resolve the vertical flow structure. Specifically, the experimental swash flows over impermeable fixed-bed beach (Kikkert et al. 2012) is simulated. With the computed vertical distribution of swash velocity that agrees with the measured data reasonably well, the bed shear velocity is estimated using three variants (Original version: calibrating both the bed shear velocity and the bed roughness height using near-bed flow velocities ; Variant I: estimate the bed roughness height empirically and calibrating only the bed shear velocity using near-bed flow velocities; Variant II: estimate the bed roughness height empirically and calibrating the bed shear velocity using depth-averaged flow velocities) of the law-of-wall method (Wilcock 1996), and the semi-theoretical formula derived from unsteadyflow equation (Mrokowska et al. 2015), as well as numerical solutions of the near-bed Reynolds shear stress from the 3D RANS model. It is shown that the Variant $I$ of the law-of-wall method give better estimations of bed shear velocity than other methods. This supports the present practice as implemented in O'Donoghue et al. (2016). More work must be done on estimation of bed shear stress/velocity.

Before a reliable and general relation for bed shear velocity is obtained, we have used three methods (calibrating a constant value for the friction factor by comparing the computed and measured swash depth and velocity, using the Swart empirical relation and using the Colebrook empirical relation) to compute bed shear stress in the depth-averaged non-capacity sediment transport model, based on which the sediment transport capacity is computed by three empirical relations (the MPM formula, the Wu formula, and the van Rijn formula). To quantify the lag between actual sediment transport 
rate and the sediment transport capacity, five existing empirical relations for the saturation length (the Armanini relation; the Jain relation; the Wu relation; the Phillips relation; the Paehtz relation) are applied. To reveal the infiltration effects, numerical cases with/without infiltration effects are conducted. In total, the above considerations give 90 numerical cases [(with/without infiltration effects: two options) * (friction factor: three options) * (sediment empirical relations: three options) * (saturation length relations: five options) for a single experimental swash process. Specifically, the experimental swash flows and sediment transport over a coarse sand, permeable, and movable beach (O'Donoghue et al. 2016) is simulated. A detailed comparison between these 90 numerical cases is conducted. The following observations are made. Firstly, including the infiltration effects can give much better predictions of swash hydrodynamics (depth, velocity and swash front positions) and sediment transport rate over a permeable bed. Concerning the present simulated experimental process, the MPM gives the best predictions of sediment transport rate among the three relations for sediment transport rate at capacity regime; and the performance of the Wu relation is better than that of the van Rijn relation.

\section{REFERENCES}

Hu, P., Li, W., He, Z., Paehtz, T., and Yue, Z. (2015). Well-balanced and flexible modelling of swash hydrodynamics and sediment transport. Coastal Engineering, ELSEVIER, VOL.96, PP 27-37.

Li, W., Hu, P., Pahtz, T., He, Z., and Cao, Z. (2017). Limitations of empirical sediment transport formulas for shallow water and their consequences for swash zone modeling. Journal of Hydraulic Research, IAHR, vol. 55, pp 114-120.

Wilcock, P. R. (1996). Estimating local bed shear stress from velocity observations. Water Resources Research, 32, 3361-3366.

O'Donoghue, T., Kikkert, G. A., Pokrajac, D., Dodd, N. and Briganti, R. (2016). Intra-swash hydrodynamics and sediment flux for dam-break swash on coarsegrained beaches. Coastal Engineering, 112, 113130.

Kikkert, G. A., O'Donoghue, T., Pokrajac, D., and Dodd, N. (2012). Experimental study of bore-driven swash hydrodynamics on impermeable rough slopes. Coastal Engineering, 60, 149-166.

Mrokowska, M. M., Rowinski, P. M., and Kalinowska, M. B. (2015). Evaluation of friction velocity in unsteady flow experiments. Journal of Hydraulic Research, 53(5), 659-669. 